\section{Scintillations from Thin Films}

Wr have investigated the emission of light pulses from thin films under $\alpha$-particle bombardment. In all cases, irrespective of the material used, light pulses were observed to be emitted from those films the thickness of which did not exceed the range of the $\alpha$-particle in the material. The following materials were included in our investigations :

Sulphur, Iodine, Carbon; Nickel Sulphate, Sodium Chromate, Stannous Chloride, Mercuric Iodide ; Aluminium, Copper, Tungsten, Iron; Nylon, Glass, Mica, Collodion.

None of these materials is normally considered to be a phosphor, the effect being entirely confined to films which are thin with respect to the $\alpha$-particle range. The efficiencies of all these films are approximately the same in respect to pulse-height and the number of counts obtained.

In the majority of cases, the substance under investigation was deposited on a $1-\mathrm{mm}$. quartz slide. This was then mounted over the cathode of a $931 \mathrm{~A}$ photomultiplier. A strong collimated polonium source was placed about $5 \mathrm{~mm}$. above the slide. Since the pulses from the multiplier are not greater than four times noise, it was found necessary to feed the signals from the multiplier direct into a suitable head amplifier with minimum capacity in the input leads. Owing to the low counting efficiency of these films, a careful and continuous check had to be made on the random count due to noise, etc. This was done by reversing the slide immediately after each reading. A quartz slide was used as it was believed that the radiation was in the ultra-violet.

For purely experimental reasons, nylon films were chosen for a more detailed examination. This included a comparison between the efficiencies of a single nylon film of $0.48 \mathrm{~mm}$. air equivalent, and a zinc sulphide screen. It was found that the counting efficiency of the nylon film was reduced by a factor of 200 and the pulse-height by about 25. A pulseheight distribution was determined (Fig. 1), but, as would be expected, no plateau was obtained.

The effect of film thickness on the phenomena was studied by bombarding a series of similar nylon films. The results are shown in Fig. 2. It appears that, by increasing the nylon by one film thickness more than the $\alpha$-particle range, the effect disappears. There was also no measurable change in the pulseheights with increasing thickness. From these considerations, we conclude that the radiation is highly



Fig. 1. Pulse-height distribution for a single nylon fllm

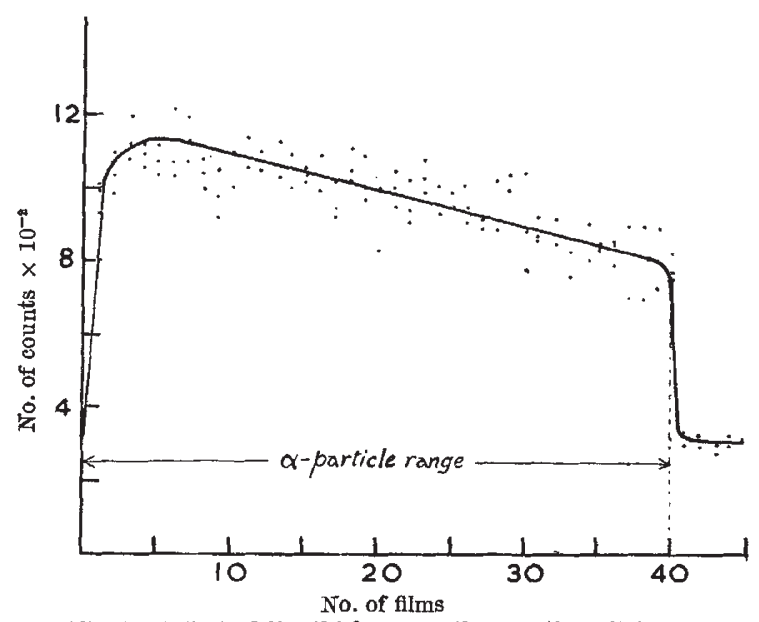

Fig. 2. Effect of fllm thickness on the counting efficiency

self-absorbent, the radiation length cannot be at most appreciably greater, and may indeed be less, than one film thickness.

No pulses were obtained when either thin films or crystals of these materials were subjected to $\beta$ - or $\gamma$-bombardment, although, in the case of nylon at least, marked fluorescence is obtained under ultraviolet irradiation.

E. W. T. RICHARDS

J. F. I. COLF

Department of Natural Philosophy,

University, Glasgow.

Nov. 27.

\section{A Sensitive Method of Recording Nuclear Induction Signals}

IN the detection of nuclear induction signals, it is usual to modulate the main polarizing magnetic field in such a way that its intensity sweeps cyclically over a small region embracing the resonance value ${ }^{1}$. If the amplified signal is fed to a cathode-ray oscilloscope in which the $X$-sweep follows the instantaneous field modulation, it gives rise to a 'blip' at a corresponding part of the trace.

A lower limit is set to visual detection in this way when the signal-level becomes comparable with that of the amplifier noise. In the usual method ${ }^{2}$ of $\theta x$ tending the sensitivity, the modulation amplitude is reduced to a small fraction of the resonance width, and the output of the detector fed to a 'lock-in' amplifier, followed by a D.c. recorder. If the signal region is traversed slowly, the recorder traces a signal the instantaneous value of which is proportional to the slope of the steady-state nuclear radiofrequency signal. By making the response-time of the output circuit sufficiently long, the signal/noise ratio may be made as high as desired-at the expense of speed of search, and subject to practical limitations of stability.

This method has the disadvantage of using the steady-stato response, which, due to thermal relaxation, is intrinsically weaker than that obtainable under suitable transient conditions. Also it requires fairly elaborate means of stabilization and control to give the necessary smooth and slow traverse.

Bloch has pointed out ${ }^{3}$ that the signal/noise ratio obtained by oscillographic presentation can be im. proved by photographic means. If an exposure is 